\title{
Recovery of 3-D shape from binocular disparity and structure from motion
}

\author{
JAMES S. TITTLE \\ The Ohio State University, Columbus, Ohio \\ and \\ MYRON L. BRAUNSTEIN \\ University of California, Irvine, California
}

\begin{abstract}
Four experiments were conducted to examine the integration of depth information from binocular stereopsis and structure from motion (SFM), using stereograms simulating transparent cylindrical objects. We found that the judged depth increased when either rotational or translational motion was added to a display, but the increase was greater for rotating (SFM) displays. Judged depth decreased as texture element density increased for static and translating stereo displays, but it stayed relatively constant for rotating displays. This result indicates that SFM may facilitate stereo processing by helping to resolve the stereo correspondence problem. Overall, the results from these experiments provide evidence for a cooperative relationship between_SFM and binocular disparity in the recovery of 3-D relationships from 2-D images. These findings indicate that the processing of depth information from SFM and binocular disparity is not strictly modular, and thus theories of combining visual information that assume strong modularity or independence cannot accurately characterize all instances of depth perception from multiple sources.
\end{abstract}

Human observers can perceive the 3-D shape and orientation in depth of objects in a natural environment with impressive accuracy. Prior work demonstrates that information about shape and orientation can be recovered from binocular disparity (e.g., Ono \& Comerford, 1977) and structure from motion (SFM) (e.g., Braunstein, 1976). However, neither SFM nor binocular disparity in isolation specifies completely both an object's shape and orientation relative to an observer. Structure from motion can provide information about object shape, but near/far relations between the object and the observer are not specified (Wallach \& O'Connell, 1953). ${ }^{1}$ Binocular disparity, on the other hand, unambiguously specifies near/far relations, but the shape of the object is only determined up to a scale factor: perceived fixation distance (Gogel, 1960; Ogle, 1953; Wallach \& Zuckerman, 1962). Because each of these sources of information specifies what the other lacks, the combination of SFM and binocular disparity could provide a more robust representation of an object than would either source alone (Richards, 1985). In addi-

This research was supported by National Science Foundation Grant BNS8819565. Portions of this research were presented at the SPIE Sensor Fusion III Conference (Tittle \& Braunstein, 1991) and at the Annual Meeting of the Association for Research in Vision and Ophthalmology (Tittle \& Braunstein, 1989). This research was completed as partial fulfillment of the requirements for the Ph.D. at the University of California, Irvine by the first author. We would like to thank Asad Saidpour for assistance in developing the stimulus displays and Joseph Lappin for helpful comments on an earlier draft of this paper. Correspondence should be addressed to J. S. Tittle, Department of Psychology, Ohio State University, 140D Lazenby Hall, 1827 Neil Avenue, Columbus, Ohio 43210 (e-mail: jtittle@magnus. acs.ohio-state.edu). tion, both sources of information (along with many others) occur together in the natural environment. Thus, it is important to understand what interaction (if any) exists in the visual processing of binocular disparity and SFM.

Recent research has considered how the human visual system combines depth information from multiple sources (Braunstein, Andersen, Rouse, \& Tittle, 1986; Bruno \& Cutting, 1988; Bülthoff \& Mallott, 1988; Dosher, Sperling, \& Wurst, 1986; Rogers \& Collett, 1989; Rouse, Tittle, \& Braunstein, 1989). Specifically, Bülthoff and Mallott discuss four types of interactions between different sources of depth information: accumulation, disambiguation, veto, and cooperation. Accumulation refers to an additive relationship between the various processes that is consistent with modularity in visual information processing (Fodor, 1983). The perceived depth from this type of interaction is a weighted summation or integration of the depth specified by each separate process. Empirical evidence for an accumulative relationship has been provided for stereo and relative brightness (Dosher et al., 1986), motion parallax and pictorial depth cues (Bruno $\&$ Cutting, 1988), and motion parallax and binocular disparity (Rogers \& Collett, 1989). A veto relationship between visual processes is one in which one source of information completely dominates others. It is also consistent with a modular conceptualization of visual depth processing. An example of a disambiguating relationship between two sources of information comes from a study by Andersen and Braunstein (1983), who used dynamic occlusion to disambiguate the order of perceived depth in orthographic SFM displays. Disambiguation of an SFM display can also result from adaptation to a stereo dis- 
play (Nawrot \& Blake, 1991). A cooperative interaction between visual processes is one in which strict modularity does not hold. Instead, information from one process facilitates the functioning of another process.

Richards (1985) has proposed a specific relationship between stereopsis and SFM that eliminates the ambiguities inherent in each source of information. According to this analysis, SFM provides information for 3-D shape and stereopsis disambiguates depth order. The derivation of 3-D shape from motion assumes parallel projection and planar motion of the points. Using this approach, Richards shows that with two stereo views of three points, the depth coordinates of those points are uniquely determined. Such a relationship between stereo and SFM would achieve shape constancy while still preserving unambiguous viewerrelative depth relations. However, one assumption of this analysis is that the magnitude of perceived depth from stereopsis and SFM depends solely on the SFM information.

Early empirical research on conflicting stereo and SFM information is consistent with Richards's (1985) theoretical analysis. Specifically, Wallach, Moore, and Davidson (1963), and Epstein (1968) found that stereopsis is recalibrated by prolonged adaptation to conflicting SFM information. In these studies, wire-frame objects were viewed through a telestereoscope that exaggerated the binocular disparities projected onto the retinae. Thus, when the objects were rotated, the binocular disparity information was consistent with an object much deeper than that indicated by the SFM information. Observers made depth judgments of static stereo displays before and after a 10-min adaptation period. Epstein found that the same amount of disparity was judged to have less depth after the adaptation period than before it. These findings seem to provide evidence that SFM dominates stereopsis, and may indeed solely determine the magnitude of perceived depth when combined with binocular disparities, as suggested by Richards. However, Fisher and Ebenholtz (1986) showed that depth aftereffects, of the type reported in the previous research, occurred even when stereo and SFM were not presented together in the adapting stimulus. Their results seem to indicate that the adaptation effects, cited as evidence for the primacy of SFM, were not due to a recalibration of stereopsis from SFM, but were caused by changes in the relationship between convergence and accommodation induced by the telestereoscope.

Lappin and Love (1992) presented empirical evidence that SFM information could be used in a curvature discrimination task, even in the presence of inconsistent disparity information. The stimuli for these experiments were ellipses slanted out of the frontoparallel plane. Observers were required to discriminate between ellipses of varying eccentricities. The results showed that discrimination accuracy was greater for rotating stimuli than for static stereo ones-a finding consistent with the data from our Experiment 1 (reported below). Lappin and Love also found that when stereo and SFM provided inconsistent information about ellipse eccentricity, the observers were still able to make accurate curvature discriminations on the basis of SFM.

In a study examining the contributions of relative brightness and stereopsis to the perception of sign of depth, Dosher et al. (1986) presented observers with perspective projections of rotating wire-frame Necker cubes. They found that direction-of-rotation judgments were primarily determined by binocular disparity. However, the relative brightness cue did have some influence on the rotation judgments, and the results were fit closely by a model in which the strength of evidence from each source of information was algebraically added. Their weighted linear summation model was similar to a more general approach for describing information integration developed by Anderson (1981, 1982).

Braunstein et al. (1986) showed that binocular disparity can disambiguate the sign of depth for computergenerated displays consisting of orthographic projections of texture elements on the surface of rotating spheres. They also found that, for opaque stimuli, dynamic occlusion information usually vetoed conflicting binocular disparity information in determining perceived sign of depth (measured by direction-of-rotation judgments). However, for transparent stimuli, direction-of-rotation judgments were primarily determined by binocular disparities. Braunstein et al. also found that 6 subjects who could not detect any targets on a static stereo test (Randot circles) were able to make accurate direction-of-rotation judgments for stereo-SFM displays on over $90 \%$ of the trials. Because the direction of rotation is inherently ambiguous in an orthographic projection, these static stereo-deficient observers must have been able to use the binocular disparity information in the stereo-SFM displays, even though they did not respond correctly to binocular disparities on a standard static stereo test.

Rouse et al. (1989) examined this effect further, and found that 7 out of 11 static stereo-deficient observers could judge, with perfect accuracy, direction of rotation with combined stereo-SFM displays similar to those used by Braunstein et al. (1986). None of the 11 observers classified as static stereo-deficient could detect even the largest disparity level $\left(500^{\prime \prime}\right)$ on the static Randot Forms Test. In contrast, the maximum disparity present in the rotating cylinder displays was only 85" (crossed or uncrossed). Thus, even though the maximum disparity in the rotating cylinder stimuli was less than one fifth the maximum disparity in the static stereo test, the majority of observers who failed to detect any static stereo stimuli could use binocular disparity information accurately in the stereoSFM display.

This last result is especially interesting, because it provides evidence for a nonmodular facilitative interaction between stereo and SFM processing. If the processing of binocular disparity and SFM is modular, then one would not expect that combining SFM and stereoscopic information would enable static stereo-deficient observers to use binocular disparities. However, the results of Braun- 
stein et al. (1986) and Rouse et al. (1989) provide evidence that this is exactly what happens when static stereodeficient observers view stereo-SFM displays.

This result suggests a facilitative interaction between stereo and SFM processing for stereo anomalous observers. However, it is not obvious that motion would facilitate stereo processing for observers with unimpaired stereoscopic depth perception. Thus, we sought to determine whether SFM and binocular stereopsis function in a modular fashion for observers with normal stereo vision, or if the processing of these two sources of information is nonmodular with SFM facilitating stereoscopic depth perception. First, we will describe a demonstration that suggests that SFM facilitates stereo processing in observers with normal stereo vision. In this demonstration, observers described the apparent depth in displays consisting of bright dots randomly positioned on the surface of simulated transparent cylinders that were either static or rotating about a horizontal axis. (Akerstrom \& Todd, 1988 , have previously reported that transparent stereo displays are particularly difficult to process.) Four experiments were conducted to examine the basis of this facilitation. In Experiment 1, we obtained magnitude estimates of depth-to-height ratio for static, rotating, and translating transparent cylinders. The translation condition was included to allow comparisons to be made between the combination of stereo with motion not involving depth change (translation perpendicular to the line of sight) and the combination of stereo with SFM (rotation about an axis other than the line of sight).

In Experiments 2, 3, and 4, we used a two-alternative forced-choice staircase method to determine the amount of stereo needed to see a fixed depth-to-height ratio. To determine if the presence of motion decreases the amount of time required to build up stereo depth, two different display durations were used in Experiment 2. The role of SFM in facilitating the resolution of the stereo correspondence process was studied in Experiments 3 and 4 by varying dot density for static, translating, and rotating transparent cylinder displays. Greater dot density should make correspondence matching more difficult by providing more false matches to any process using a nearest neighbor criterion. If SFM facilitates stereo processing by helping to solve stereo correspondence matching, we would expect greater facilitation with greater texture density.

\section{Demonstration}

As we stated earlier, there are many possible interactions between SFM and binocular disparity information. These include cooperative, competitive, and completely modular relationships between the two processes. A demonstration developed independently by Tittle and Braunstein (1989) and Pong, Kenner, and Otis (1989) will help illustrate the potentially complex nature of this interaction.

Tittle and Braunstein (1989) used computer-generated stereo displays consisting of 1,000 bright dots projected orthographically onto the surface of a transparent cylinder. The cylinder rotated about a horizontal axis and, thus, the SFM information was consistent with an object equal in depth and height. The original stereo displays were presented as red-blue anaglyphs, but the observations were later confirmed by using side-by-side monochromatic images fused with a mirror stereoscope. The depth-to-height ratio indicated by disparity could be varied independently of the ratio indicated by SFM, which was always $1: 1$. In contrast, the binocular disparities were consistent with an object with a depth-to-height ratio of $3: 1$.

When the cylinder displays were rotating, the observers reported seeing an object with a depth-to-height ratio greater than 1:1. This implies that the perceived depth in these displays results at least partially from the binocular disparity information, because the SFM information indicated an object that was equal in depth and height (and a depth-to-height ratio greater than $1: 1$ is virtually never reported in monocular viewing of these displays). However, when the motion in the display was halted, leaving only binocular disparities specifying depth, the observers reported that the displays appeared to have little or no depth.

Two aspects of this demonstration provide evidence relevant to the question of an interaction between the processing of SFM and binocular disparity. First, when both sources of information were present, the judged depth was greater than that indicated by SFM alone. This observation is inconsistent with Richards's (1985) suggestion that the perceived shape should depend solely upon SFM, with only the sign of depth determined by binocular disparities. Second, even though binocular disparities were present in the static displays, the observers were apparently unable to use this information. Thus, the SFM, or at least some aspect of the motion, in the rotating displays facilitated the use of binocular disparities.

\section{EXPERIMENT 1}

There are a number of possible reasons why the perceived depth appeared greater for rotating transparent cylinders than it did for static ones. First, the correspondence problem is particularly severe in transparent displays, due to the possibility of incorrectly pairing front surface texture elements with back surface elements (Akerstrom \& Todd, 1988). To the extent to which local matches are determined by a nearest neighbor criterion, these incorrect pairings are most likely to occur when a dot has a potential match closer to it than the correct match. Thus, the incorrect pairing will generally result in less disparity than the correct pairing of points and we would predict that judged depth would decrease as the number of false matches between dots in the left and right images increased. In the rotating display, the front and back texture elements differ in the signs of their velocities, and this difference could be used to eliminate potential false matches between elements on the two surfaces. Support for this explanation comes from a demonstration similar to that described above, which was conducted with an opaque version of the rotating cylinder stimulus. When only the front half of the cylinder was visible, the ob- 
servers reported less of a difference between the static and dynamic displays than they had reported for the transparent displays. For the static versions of the opaque displays, the correspondence problem was possibly not severe enough to require the additional information provided by SFM.

Another possible explanation for the observation that perceived depth was greater for the rotating transparent displays than for the static transparent displays is that texture elements with particularly large disparities could not be fused in the static transparent displays. The elements with the largest disparities, which were located where the simulated depth separation between the front and back surfaces was greatest, may have appeared diplopic. Near the top and bottom of the display, the disparity approached zero, so we would not expect fusion problems in those areas. Greater perceived depth in the rotation condition would be expected if rotation aided fusion of texture elements with large disparities. Fusion of large disparity elements in the rotation condition might have been facilitated by the reduction in the number of false correspondence matches that would occur when front and back elements were moving in opposite directions, as discussed above.

A third possible explanation for the demonstration result is that the SFM process provides information about surface curvature and relative separation of the front and back surfaces of the object, thereby facilitating interpolation of a curved surface between the visible feature points. In addition, the greater perceived depth for the rotating displays than the static displays could have resulted from differences between the processing of constant versus dynamic disparity. This interpretation would be consistent with the evidence presented by Regan and his collaborators for the existence of two separate disparity-sensitive subsystems in the visual system: one sensitive to static or position disparity, and the other to dynamic or motion disparity (see, e.g., Regan, Beverley, \& Cynader, 1979). ${ }^{2}$

With these issues in mind, we conducted an experiment to address the following question about the interaction between SFM and binocular disparity information: Will any display with opposite sign velocities associated with the front and back surfaces of an object facilitate stereo processing, or does the previously observed interaction between motion and disparity processing depend upon the presence of SFM? To address this question, an additional type of display was generated that provided opposite sign velocities between the front and back texture elements, but contained no SFM information. This display consisted of an orthographic projection of a "split" cylinder translating orthogonal to the line of sight. All of the elements on the surface of the cylinder translated with equal magnitude velocity. However, the elements on the front and back surfaces differed in the sign of their velocities. Because this was an orthographic projection, the motion provided no information about relative depth or surface curvature, and thus allowed us to study the facilitation of stereo processing solely on the basis of the opposite sign velocities. Although these displays also contained a static texture gradient, the results reported below for the monocular viewing condition show that this was not effective in indicating the cylindrical shape. The observers were asked to make relative depth-to-height judgments for these split-cylinder displays, for rotating cylinder displays similar to those used in the demonstration, and for static stereo cylinders.

\section{Method}

Subjects. The subjects for this experiment were the first author and 3 graduate students from the University of California, Irvine. The latter 3 subjects were paid for their participation and were naive to the purpose of the experiment. The 3 naive subjects were unaware of the levels of simulated stereo and motion depth.

Design. Three variables were examined: (1) binocular disparity, in which simulated objects had depth-to-height ratios of $1: 1,2: 1$, and $3: 1 ;(2)$ display motion, which was either rotation, translation, or static; and (3) initial direction of motion, which was either right/left for the translating displays or clockwise/counterclockwise for the rotating displays. A monocular viewing condition was also included so that comparisons could be made between the amount of depth perceived from motion information alone and that resulting from the combination of motion and stereopsis.

Stimuli and Apparatus. The stimuli were 9 (3 motion types $\times$ 3 disparity levels) computer-generated displays consisting of 1,000 bright dots randomly positioned on the surface of a transparent cylinder and viewed against a dark background (see Figure 1). For the rotation condition, the displays simulated a transparent cylinder rigidly rotating, with a constant angular velocity of $6.5 \% \mathrm{sec}$, about a horizontal axis. Each display rotated continuously, either clockwise or counterclockwise, for the duration of a trial. The displays for the translation condition simulated a transformation in which the front and back halves of a cylinder translated along the horizontal axis with equal magnitude but opposite sign velocities. Unlike the rotation condition, the direction of motion was not constant, but cycled at $1 \mathrm{~Hz}$. For example, at the beginning of a translation trial, the observer would see a display in which the front surface was moving to the right and the back surface was moving to the left. After $1 \mathrm{sec}$, the front surface would begin moving to the left and the back surface toward the right. The left and right boundaries of these displays were clipped so that the display would maintain a constant size throughout the motion sequence. Thus, as a dot moved beyond the far right or left edge of the display, it would disappear. As dots disappeared at one edge, other dots would appear at the opposite edge.

The two views of the stereo pair were $16.5 \mathrm{~cm}^{2}$ in size, and subtended a visual angle of $5.4^{\circ}$. The center-to-center separation of the stereo pair was $20 \mathrm{~cm}$. The stimuli were presented on an IMI 455 vector graphics system with $4,096 \times 4,096$ resolution at a frame rate of $17 \mathrm{~Hz}$. For the rotating displays, this resulted in a maximum texture element velocity of $0.3 \% / \mathrm{sec}$, and for the translating displays a maximum velocity of $0.34 \% \mathrm{sec}$. The two views of the stereo
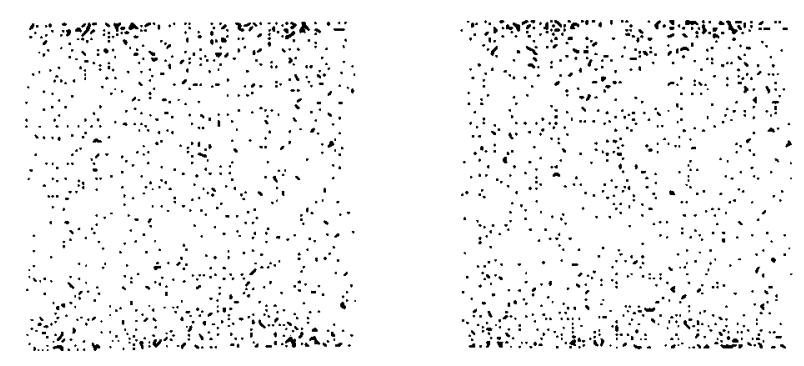

Figure 1. Static view of cylinder stereogram used in Experiment 1. (The actual displays were white dots on a dark background.) 
pair were observed through a mirror stereoscope that contained a prism in front of the subject's left eye to aid convergence. The three maximum disparity levels simulating objects with depth-to-height ratios of $1: 1,2: 1$, and $3: 1$, were $5^{\prime} 30^{\prime \prime}, 11^{\prime}$, and $17^{\prime}$ of arc crossed and uncrossed disparity, respectively.

Procedure. The subjects were run individually. They were seated $174 \mathrm{~cm}$ away from the CRT, and viewed the displays through the mirror stereoscope. The subjects were told that they would be viewing a series of displays of bright dots on the surface of a transparent object, and that their task was to make a verbal judgment of the depth-to-height ratio for each display. In addition, they were instructed, for the motion displays, to indicate the direction in which the dots on the front surface of the object were moving-up or down for the rotation displays and right or left for the translation displays. This provided a way of determining whether the subjects were properly fusing the stereo images. After the instructions were read, the subject viewed one block of 9 practice trials and two experimental blocks consisting of 18 trials each. The subjects were allowed to view each display for as long as they felt necessary to make the depth-to-height ratio judgment.

\section{Results and Discussion}

The average depth-to-height judgments for the nine binocular conditions ( 3 motion types $\times 3$ disparity levels) and the three monocular conditions are shown in Figure 2. A 3 (motion) $\times 3$ (disparity) $\times 2$ (initial motion direction) factorial analysis of variance (ANOVA) was performed on the depth-to-height judgments for the binocular conditions. Both the main effects of display motion $[F(2,6)=8.6, p<.05]$ and binocular disparity $[F(2,6)$ $=29.3, p<.01]$ were significant. From Figure 2 it can be seen that, for all binocular disparity levels, judged depth was greatest for the rotation displays, intermediate for the translation displays, and smallest for the static displays. In addition, judged depth, in both motion conditions, varied with binocular disparity. This provides further evidence that perceived depth in SFM-stereo displays depends at least partially on the available binocular disparity information.

For the static displays at the two lower binocular disparity levels (simulating depth-to-height ratios of $1: 1$ and 2:1), judged depth increased with simulated stereo depth.

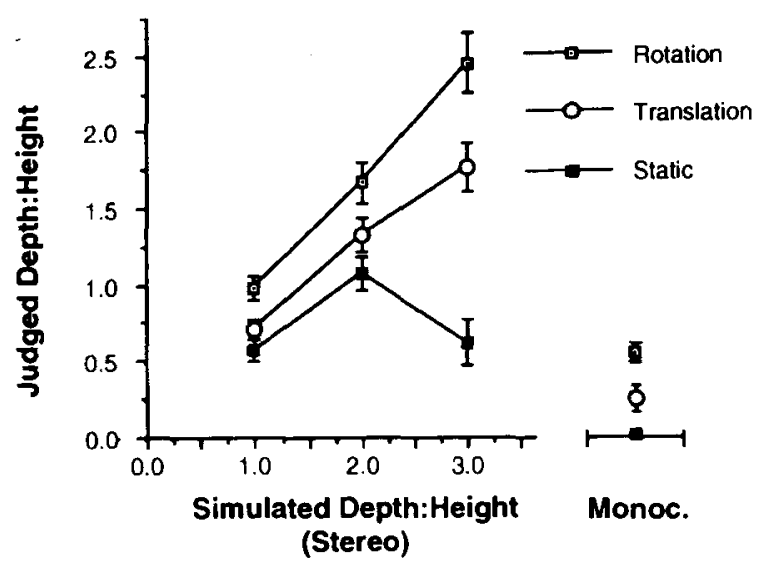

Figure 2. Judged depth-to-height ratio as a function of display motion and simulated stereo depth. Error bars are plus and minus one standard error.
However, for the highest binocular disparity level, judged depth dropped off considerably and was approximately equal to that for the minimum disparity level. This effect was reflected in the analysis as a significant interaction between motion type and disparity level $[F(4,12)=4.0$, $p<.05]$. The facilitating effect of motion can most readily be seen for the maximum binocular disparity conditions. When binocular disparity simulated a depth-to-height ratio of $3: 1$, the rotating displays were judged to have a depth approximately 5 times greater than the static displays. Not all of this difference can be attributed to SFM, however, because the maximum disparity level translating displays were judged to have approximately 3.7 times more depth than the equivalent static displays. However, a post hoc test (Tukey's HSD) indicated that, for the maximum binocular disparity level, judged depth for the rotation displays was significantly greater than for the translation displays $(p<.05)$.

The significant decrease in judged depth at the highest disparity level in the static condition is consistent with Akerstrom and Todd's (1988) results with high-disparity transparent stereograms. Although the maximum disparity for our displays may have been outside the foveal fusional limits, the observers did not report that the entire display appeared diplopic. Our observers did report qualitative differences between the appearance of the center and the top and bottom edges of the static condition displays. Specifically, some observers noted that they could see a smooth surface near the top and bottom of the display, but in the center region the dots appeared to randomly fill a volume, rather than define a near and far surface. The observers also reported that some dots in the central region of the display appeared diplopic in the static condition. These reports are consistent with the magnitude of disparities in our displays. Disparity reached a maximum at the center of the display and smoothly approached zero toward the top and bottom of the display. Thus, assuming that the probability that a point's nearest neighbor will be an incorrect match increases as the disparity of the correct match increases, we would expect that more correspondence mismatches would occur in the central region of our transparent cylinder stimuli.

The results of the direction-of-motion judgments for the translation and rotation conditions indicated that the observers were accurately using disparity information for all three disparity levels in the motion conditions. For the rotation displays, all the observers indicated the direction of motion accurately on every trial. Because these were parallel projections, the motion did not provide information about near/far relationships and thus binocular disparity was the only information available to determine the direction of rotation. Our observers were only slightly less accurate in the translation condition; 2 observers accurately indicated the direction of motion for all trials and the other 2 each made one error in 48 trials (both at the highest disparity level). Thus, despite the large disparities used in our displays, the observers were able to accurately use binocular disparities in almost all of the motion condition trials. 
Figure 2 also shows the results for monocular viewing of the rotation, translation, and static displays. A 3 (rotation, translation, static) $\times 2$ (initial motion direction) ANOVA was performed on the average depth-to-height judgments for the monocular conditions. Only the main effect of motion type was significant $[F(2,6)=42.2, p<$ $.01]$. The greatest depth-to-height ratios were judged in the rotation displays $(0.61)$, followed by the translation displays $(0.17)$. Not surprisingly, the static displays were judged to be relatively flat $(0.04)$.

These data suggest that motion facilitates the processing of binocular disparity in transparent stereo displays. As can be seen in Figure 2, the depth judgments for the translation and rotation conditions were significantly greater than for the static conditions. However, perhaps more importantly, the rotation displays, which provided SFM information, were judged to have greater depth than the translation displays. This pattern of results demonstrates that motion facilitates stereo processing, but it also indicates that SFM might provide additional facilitation. However, an orthographic projection of pure translation provides no relative depth information, and thus another interpretation of the difference between the two motion conditions is that judged depth for the stereo motion displays depends on a combination of the depths perceived from the disparity and motion information. This possibility is considered in Experiment 4.

The conclusion that perceived object shape in stereoSFM displays is not based solely on motion information gains additional support from the finding that the judged depth-to-height ratios for the rotating cylinders increased monotonically with binocular disparity. Indeed, depth judgments for the three disparity levels, which simulated objects with depth-to-height ratios of $1: 1,2: 1$, and $3: 1$, was reasonably accurate: $0.98,1.7$, and 2.5 , respectively. The greater underestimation in judged depth with greater simulated stereo depth could have resulted from the fact that the SFM information for all three disparity levels simulated objects that were equal in depth and height. Thus, only for the lowest disparity level would the stereo and SFM information have provided consistent information about object shape.

\section{EXPERIMENTS 2A AND 2B}

The following experiments were designed to examine whether motion facilitates stereo processing by aiding in the solution of the stereo correspondence problem, and whether SFM provides greater stereo facilitation than translation. Experiment 2 examined the possibility of a relationship between facilitation and stimulus duration. Experiments 3 and 4 used texture density variations to study the role of stereo correspondence in the facilitation of stereo processing by motion.

One way that motion could facilitate stereo processing would be to decrease the amount of time it takes to resolve the stereo correspondence problem. To test this possibility, in Experiment 2 the displays were presented for two different durations (750 and 3,000 msec). These durations were selected on the basis of pilot studies, which indicated that the task was too difficult in all conditions with shorter durations. Previous research (Akerstrom \& Todd, 1988) has shown that accuracy of relative depth judgments with static transparent stereograms improved as display durations increased from 100 to $1,000 \mathrm{msec}$. For the static condition, we expected that judged depth would be less for the short than for the long stimulus duration because of insufficient time to complete the stereo correspondence process. However, if motion facilitates stereo processing by providing a faster solution of the correspondence problem, judged depth for the motion conditions should be relatively constant for the two stimulus durations. Thus, we expected to find an interaction between stimulus duration and display motion.

In the first experiment, judged depth was greater for the rotation displays than it was for the translating displays. However, the motion was in the vertical direction for the rotation displays and the horizontal direction for the translating displays, so the difference in judged depth could have been due to the different motion directions. To rule out this possible interpretation, the orientation (horizontal/vertical) of the stimulus was also varied in Experiment 2. Because of the short durations used in this experiment, a new response method was employed. An interleaved staircase paradigm was used to determine how much stereo depth an observer required in order to see a cylindrical object with a depth-to-height ratio of either 1:1 (Experiment 2A) or 2:1 (Experiment 2B). This method was similar to a technique previously used by Johnston, Landy, Cumming, and Maloney (1991). Our objective was to determine whether more disparity is needed to achieve a particular perceived depth-to-height ratio in the static conditions than in the motion conditions, and whether this difference is greater with shorter durations (and with higher densities in Experiments 3 and 4). For this reason, the depth-to-height ratio simulated by SFM (1:1) was constant across durations and across density conditions and the stereo and SFM information may have provided conflicting indications of the amount of depth in the display, especially when the subjects were asked to indicate whether the depth-to-height ratio was greater or less than 2:1. The present experiments were not intended to determine the relative weights of the two sources of information, which would require independently varying both sources (see, e.g., Johnston et al., 1991; Tittle \& Braunstein, 1991, Experiment 2).

\section{Method}

Subjects. Four graduate students, naive to the purpose of the experiment, and the first author participated in Experiment $2 \mathrm{~A}$. Four of the subjects who participated in Experiment $2 \mathrm{~A}$, including the author, also participated in Experiment $2 \mathrm{~B}$. The data from 1 additional observer in Experiment $2 \mathrm{~B}$ was not included in the analysis because his staircase sequences failed to converge for most conditions. All the observers participated in Experiment 2A first. The naive observers were paid for their participation in both experiments.

Design. The design for both experiments was the same, and consisted of the following independent variables: (1) display motion (static, translation, or rotation), (2) stimulus duration ( 750 or 3,000 
msec), and (3) cylinder orientation (horizontal or vertical). The dependent measure was the amount of disparity needed to see a depthto-height ratio of 1:1 for Experiment $2 \mathrm{~A}$ and 2:1 for Experiment 2B. We will refer to these as the point of subjective equality (PSE) for a 1:1 depth-to-height ratio ( $\mathrm{PSE}_{1: 1}$ ) and a 2:1 depth-to-height ratio (PSE $2: 1$ )

Stimuli and Apparatus. The stimuli were similar to those used in Experiment 1, except that the number of dots was increased from 1,000 to 1,100 . The maximum disparity of dots on the surface of objects with depth-to-height ratios of $1: 1$ and $2: 1$ were $4.30^{\prime}$ and $8.65^{\prime}$ of arc, respectively. The displays were presented on an IMI 455 vector graphics system, displayed at a rate of $18 \mathrm{~Hz}$, and viewed through a dual-channel mirror stereoscope. Unlike the stereoscope used in Experiment 1, this stereoscope did not contain a prism. Instead, the two mirrors in each channel of the stereoscope were fixed at a $45^{\circ}$ angle to the CRT and the amount of convergence needed to fuse a stereo pair was adjusted by changing the separation of the two images. Center-to-center image separation for Experiments $2 \mathrm{~A}$ and $2 \mathrm{~B}$ was $20 \mathrm{~cm}$. The convergence angle for this image separation was consistent with that needed to view an object at $200 \mathrm{~cm}$. The observers were seated approximately $185 \mathrm{~cm}$ away from the CRT, but the stereoscope added an additional $15 \mathrm{~cm}$ so that the accommodative viewing distance was $200 \mathrm{~cm}$.

Procedure. Each subject participated individually and was instructed to judge whether the depth-to-height ratio for each display was greater than 1:1 (Experiment 2A) or 2:1 (Experiment 2B). The experiment consisted of one practice and three experimental sessions; no more than two sessions were run on the same day. Each session consisted of eight interleaved staircases. The orientation and duration variables were run within a session, but the display motion variable was run between sessions. One staircase consisted of 27 trials, and began with the depth of the object either above or below the target depth-to-height ratio $(1: 1$ or $2: 1)$.

A trial began with the appearance of a fixation box, the same size as the cylinder stimulus, which was visible for $1 \mathrm{sec}$, followed by the random-dot cylinder stimulus. After the cylinder disappeared, a cursor appeared in the middle of another fixation box. A long line appeared above the cursor and a short line below it, and the subject was instructed to move the cursor toward the long line if the depth-to-height ratio of the cylinder was greater than 1:1 (Experiment $2 \mathrm{~A}$ ) or $2: 1$ (Experiment $2 \mathrm{~B}$ ), or move it toward the short line if the depth-to-height ratio was less than the target value. If the subject indicated that the display was shallower than the target depth-to-height ratio, the disparity was increased by $0.5^{\prime}$ of arc for the next trial in that particular staircase. The disparity was decreased by the same amount if the subject indicated that the display was deeper than the target depth-to-height ratio.

\section{Results and Discussion}

The estimate of the stereo $\mathrm{PSE}_{1: 1}$ or $\mathrm{PSE}_{2: 1}$ for each staircase was determined by computing the mean of all the maximum and minimum values in the staircase sequence. Stereo points of subjective equality were converted from binocular disparity to depth-to-height ratios by assuming an interocular separation of $6.5 \mathrm{~cm}$ and a perceived distance of $200 \mathrm{~cm}$.

In Experiment 2A, the observers were instructed to indicate whether a cylindrical object had a depth-to-height ratio greater or less than $1: 1$. If motion facilitates or combines with binocular disparity, then it should take less disparity to see a 1:1 depth-to-height object for a motion display than for a static display. ${ }^{3}$ The mean PSE $_{1: 1}$, plotted as a depth-to-height ratio, follows this pattern exactly (see Figure 3). A 3 (display motions) $\times 2$ (durations) $\times 2$ (orientations) ANOVA was conducted on the $\mathrm{PSE}_{1: 1}$

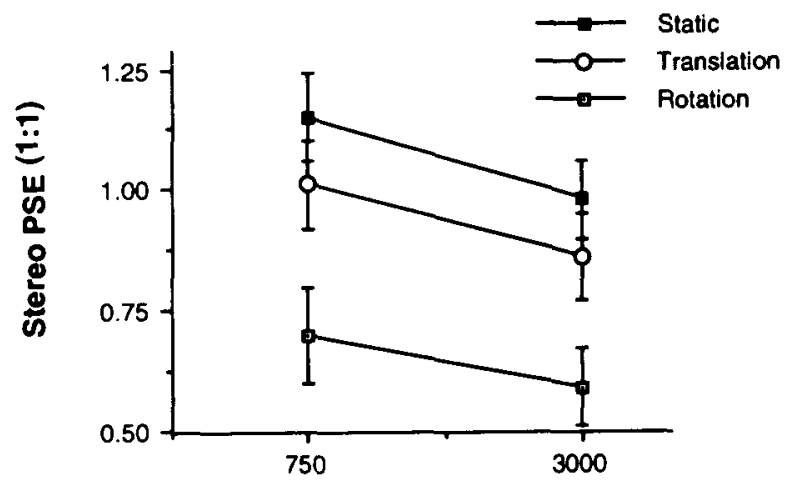

Stimulus Duration (msec)

Figure 3. Stereo PSE $1: 1$ (averaged across cylinder orientation) as a function of stimulus duration and display motion in Experiment 2A. Error bars are plus and minus one standard error.

values shown in Figure 3 . This analysis indicated that the effects of motion $[F(2,8)=9.8, p<.01]$ and duration $[F(1,4)=7.5, p<.05]$ were significant. However, the interaction between display motion and duration was not significant $[F(2,8)=2.2, p>.05]$. No other main effects or interactions were significant. Figure 3 shows that less binocular disparity (in units of simulated depth:height) was required to see the target depth for the rotation (.65) displays than for either the translation (.94) or static (1.07) displays. A post hoc test (Tukey's HSD) indicated a significant difference at the .05 level between the rotation condition and the translation and static conditions. However, the difference between the translation and static conditions was not significant.

In the present investigation, we were concerned with the interaction of motion and binocular disparity in determining the magnitude of perceived depth. It is also possible to interpret the PSE $1: 1$ results in terms of accuracy relative to the simulated stereo depth. If viewed in this way, our data indicate an overestimation of depth for both motion conditions, with the static condition providing the most accurate judged depth. One difficulty with interpreting these data on the basis of accuracy is that, in our reduced viewing conditions (darkened room and viewing through a two-channel mirror stereoscope), we cannot assume that the observer's perceived distance to the monitor was veridical.

The lack of a significant interaction between display motion and stimulus duration indicates that motion might not facilitate stereo processing by enabling a faster solution of the correspondence problem, at least for the range of durations studied. However, the maximum disparity simulating a 1:1 depth-to-height ratio was well below the disparity level that showed the largest facilitation effect in Experiment 1 . It may be that temporal facilitation will only be observed for larger disparities. Thus, in Experiment 2B, the observers were instructed to indicate whether a cylindrical object had a depth-to-height ratio greater or less than $2: 1$. A 3 (display motions) $\times 2$ (durations) $\times$ 
2 (orientations) ANOVA was conducted on the average $\mathrm{PSE}_{2: 1}$ (see Figure 4). This analysis indicated that the effects of motion $[F(2,6)=10.1, p<.05]$ and duration $[F(1,3)=16.3, p<.05]$ were significant. A post hoc test (Tukey's $H S D$ ) indicated that the PSE $_{2: 1}$ for only the rotation $(2.00)$ and static $(2.65)$ conditions were significantly different at the .05 level. As in Experiment $2 \mathrm{~A}$, the interaction between display motion and duration was not significant $[F(2,6)=0.9, p>.05]$. No other main effects or interactions were significant.

The data from Experiments $2 \mathrm{~A}$ and $2 \mathrm{~B}$ are consistent with the finding in Experiment 1 that judged depth for an SFM-stereo display is greater than judged depth for translation stereo and static stereo displays. In addition, the effect of cylinder orientation was not significant. Thus, the difference between the translation and rotation displays in the first experiment was probably not due to the difference in the direction of motion for these two conditions. However, in neither Experiment 2A nor 2B did we observe an interaction between the stimulus duration and the type of display motion. This suggests that the facilitation of binocular disparity processing in the motion conditions was not the result of a decrease in the time required for the stereo matching process.

\section{EXPERIMENT 3}

Although the data from Experiment 2 indicate that motion does not provide temporal facilitation of stereo processing, motion may still facilitate the solution of the correspondence problem. One way such facilitation could occur would be in terms of the reduction of the number of false matches that occur in the correspondence process. To examine this possibility, we varied the level of texture density in Experiments 3 and 4. Akerstrom and Todd (1988) found that increasing the density of static transparent stereograms led to both a decrease in the accuracy of relative depth judgments and an increase in the

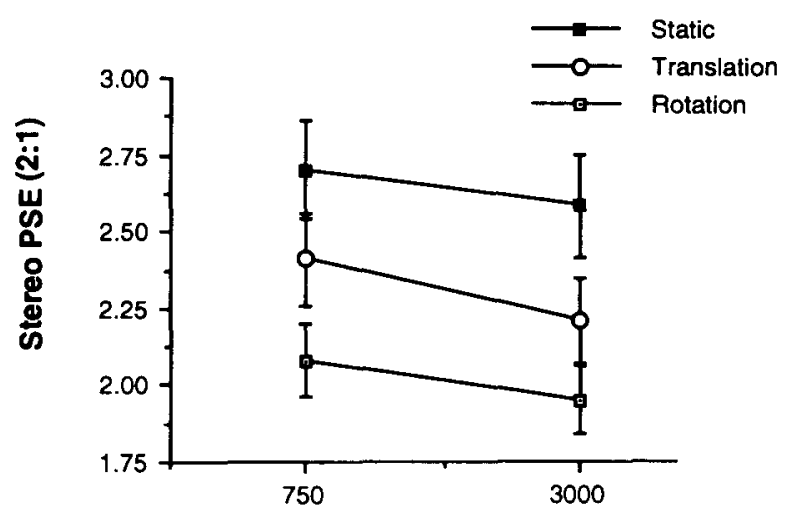

Stimulus Duration (msec)

Figure 4. Stereo PSE $_{2: 1}$ (averaged across cylinder orientation) as a function of stimulus duration and display motion in Experiment $2 B$. Error bars are plus and minus one standard error. time required to make those judgments. Stimulus duration was also manipulated in the present experiment to determine whether there was an interaction between display density and the time required to process binocular disparities in transparent stereo displays.

Because the texture elements in all of the experiments presented here were the same in brightness and shape, no static information exists that might distinguish among the texture elements and aid in the solution of the stereo correspondence problem. Thus, the visual system might initially rely on a nearest neighbor criterion to match points in the right and left images. ${ }^{4}$ For transparent displays, it is possible for points at different depths to project to similar 2-D coordinates, and mismatches can occur. As the number of points in the display increases, the probability that points from different depths will have similar 2-D coordinates also increases. Thus, increasing the number of points in a stereo display should lead to an increase in the number of incorrect stereo matches. On the basis of a nearest neighbor type matching rule, we would predict that the incorrect matches will usually have less disparity than the correct matches. Consequently, as the number of incorrect matches increases, the average disparity in the display should decrease. If we assume a monotonic relationship between judged depth and disparity in our stimuli, we would expect to find a decrease in judged depth, reflected by an increase in $\mathrm{PSE}_{2: 1}$.

\section{Method}

Subjects. The first author and 2 other observers from the previous experiment participated in Experiment 3 . These 2 additional observers were naive to the purpose of the experiment and were paid for their participation.

Design. Three variables were examined in this experiment: (1) display motion (static or rotation), (2) texture element density (200,528, or 1,400 points), and (3) stimulus duration (750 or $3,000 \mathrm{msec}$ ). Subjects was also included as a variable in the design and so the within-cell variance was computed from the six replications per condition. The dependent measure was the PSE $2: 1$.

Stimuli and Apparatus. The stimuli and apparatus were similar to those used in Experiment 2, except that either 200, 528, or 1,400 points were projected on the cylinder surface.

Procedure. The procedure was similar to that used for Experiment 2. Each session consisted of two blocks, each containing four interleaved staircases. Within a block, the four staircases all had the same density level and came from the same display motion condition.

\section{Results and Discussion}

A 3 (subjects) $\times 2$ (display motions) $\times 3$ (densities) $\times$ 2 (stimulus durations) ANOVA was conducted on the average values of $\mathrm{PSE}_{2: 1}$. The effects of display motion $[F(1,15)=49.6, p<.01]$, density $[F(2,30)=11.2, p<$ $.01]$, and duration $[F(1,15)=272.3, p<.01]$ were all significant. As in the previous experiments, the $\mathrm{PSE}_{2: 1}$ was higher for static (2.82) than for rotation (2.53) displays. In addition, the mean $\mathrm{PSE}_{2: 1}$ values for the low(2.5), medium- (2.65), and high- (2.8) density levels indicate that increasing density caused some disruption of stereo processing. A post hoc test (Tukey's HSD) revealed that the average $\mathrm{PSE}_{2: 1}$ for the low-density condition was significantly different from the medium- and 

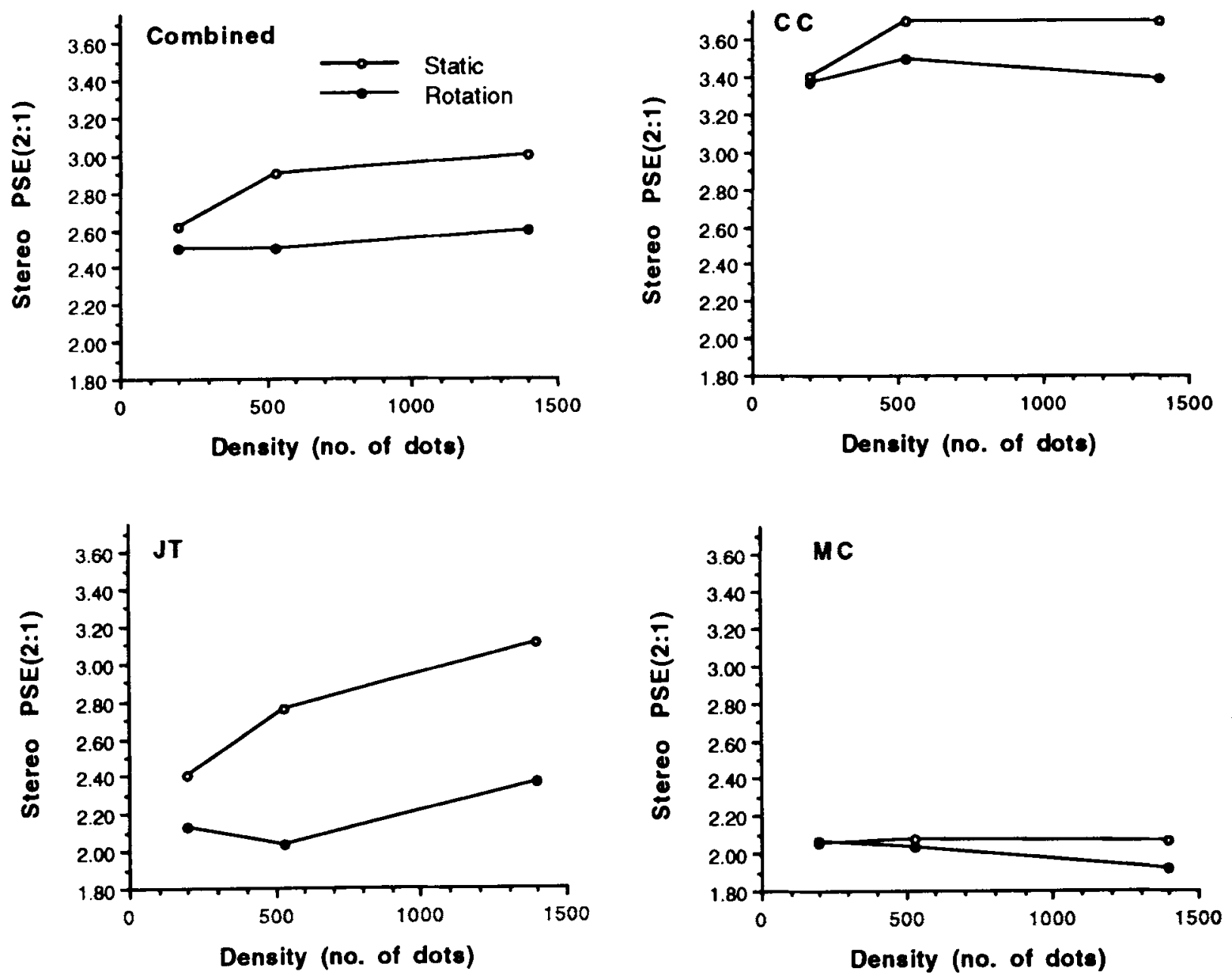

Figure 5. Stereo $\mathrm{PSE}_{2: 1}$ as a function of dot density and display motion (static or rotation) in Experiment 3.

high-density conditions at the .05 level. However, as can be seen in Figure 5, the disruptive effect of increasing density was reduced for the rotation displays. The differential effect of density on the static and rotation conditions was shown in the analysis as a significant display motion $\times$ density interaction $[F(2,30)=6.2, p<.01]$. Subjects was also included as a variable in the design, and there was a significant main effect of this variable $[F(2,15)=112.1, p<.01]$. In addition, the interaction between subjects and display motion $[F(2,15)=15.5$, $p<.01]$, duration $[F(2,15)=11.4, p<.01]$, and density $[F(4,30)=10.1, p<.01]$ were all significant. No other interactions were significant.

The reduction in the disruptive effect of high densities for rotation displays supports the hypothesis that motion facilitates stereo processing. An additive combination of depth from SFM and stereo would have been expected to generate parallel curves in Figure 5. However, the difference between the stereo PSE $2: 1$ values for the static and rotation conditions was greater for the high-density than it was for the low-density condition. This is consistent with a cooperative interaction between motion and stereo processing, with greater facilitation of stereo by SFM occurring when the stereo matching problem is more difficult.

\section{EXPERIMENT 4}

The results from Experiment 3 provide support for the hypothesis that motion facilitates stereo processing by helping to resolve the stereo correspondence problem. However, what still needs to be determined is whether or not SFM provides greater facilitation of stereo processing than other texture element motions that provide opposite sign velocities for front and back surfaces. In Experiment 1, we found some stereo facilitation with translating cylinders with opposite sign velocities, but significantly less depth was reported for these displays than for the rotating cylinders. However, it is not clear whether the difference between rotating and translating displays occurred because of greater facilitation of disparity processing by SFM or because of the greater monocular depth perceived in the SFM displays. To address this question in Experiment 4, 
we obtained $\mathrm{PSE}_{2: 1}$ estimates for high-and low-density stimuli with rotation, translation, and static displays. If facilitation of stereo processing is equivalent for SFM and translation, the difference between the $\mathrm{PSE}_{2: 1}$ values of these two conditions should not depend on the density level. If the greater judged depth found for SFM as compared with translation in Experiment 1 was due to differences in the depth implied by SFM alone as compared with translation alone, we would expect the SFM and translation curves in the present experiment to be separated by a constant amount, regardless of the density level. If, on the other hand, SFM were more effective than translation in aiding in the solution of the stereo correspondence problem, we would expect to find the difference between the $\mathrm{PSE}_{2: 1}$ for rotation and translation to be greater for the high-density condition, for which the correspondence problem should be more difficult.

\section{Method}

Subjects. The first author and 2 observers who were naive to the purpose of the study participated in this experiment. None of the naive observers had participated in any of the previous experi- ments. Both naive observers were paid for their participation. One additional observer completed the entire experiment, but her data was not included in the analysis because her staircase sequences failed to converge in most of the conditions. Another observer completed two practice sessions, but was not run in the experiment because none of his staircase sequences converged

Design. Two variables were examined in this experiment: (1) display motion (static, translation, and rotation), and (2) texture element density ( 200 or 1,300 dots). Subjects was also included as a variable in the design, and so the within-cell variance was computed from the six replications per condition. The dependent measure was the $\mathrm{PSE}_{2: 1}$.

Stimuli and Apparatus. The stimuli and apparatus were similar to those used in Experiment 2, except that the larger density was reduced to 1,300 in all conditions because of technical limitations in displaying the translation stimuli.

Procedure. The procedure was similar to that used for Experiment 2, except that all displays were visible for $1 \mathrm{sec}$. Each session consisted of two blocks, each containing six interleaved staircases. Within a block, the six staircases all had the same density level and came from the same motion condition.

\section{Results and Discussion}

The average PSE $_{2: 1}$ values for both density levels of the static, translation, and rotation conditions can be seen
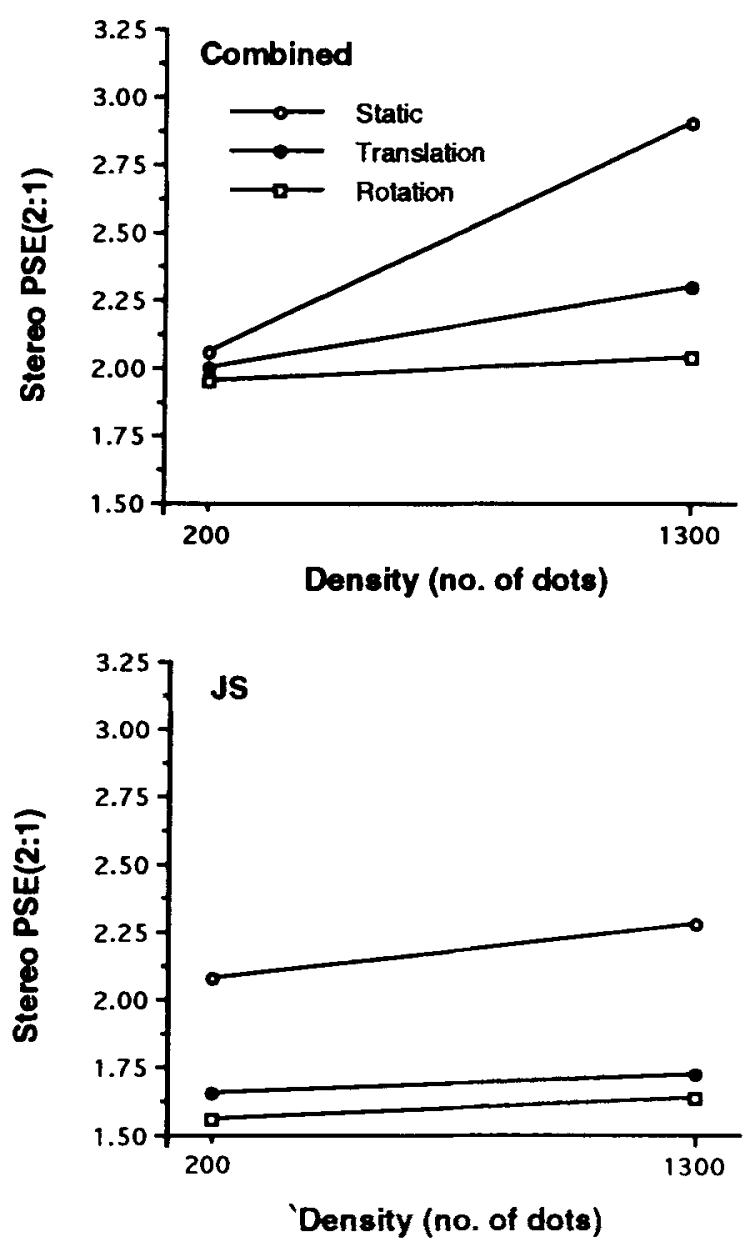
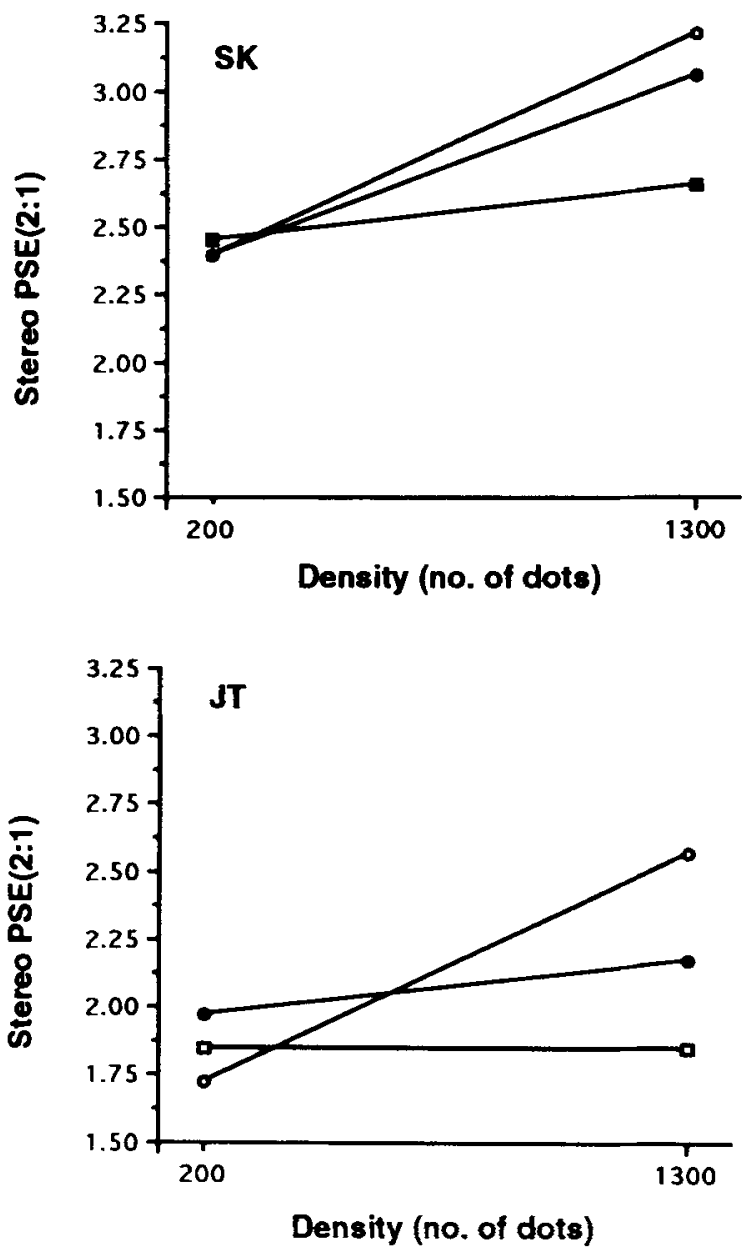

Figure 6. Stereo $\mathrm{PSE}_{2: 1}$ as a function of dot density and display motion (static, translation, or rotation) in Experiment 4. 
in Figure 6. A 3 (subjects) $\times 3$ (display motions) $\times 2$ (densities) ANOVA was conducted on the average PSE $_{2: 1}$. The main effects of display motion $[F(2,30)=37.0, p<$ $.01]$ and density $[F(1,15)=182.6, p<.01]$ were significant. Post hoc tests (Tukey's $H S D$ ) indicated that the PSE $_{2: 1}$ values for the static (2.4), translation (2.11), and rotation (1.9) conditions were all significantly different from each other at the .01 level. More importantly, the interaction between display motion and disparity was also significant $[F(2,30)=23.0, p<.01]$. In addition, the interactions between subjects and display motion $[F(4,30)$ $=5.4, p<.01]$, subject and density $[F(2,15)=28.0$, $p<.01]$, and subject, display motion, and density $[F(4,30)=5.04, p<.01]$ were all significant.

These results quite clearly indicate that both translation and rotation facilitate stereo processing. This can most easily be seen by considering the difference between the three motion conditions at the low- and high-density levels. For the low-density condition (200 points), post hoc tests (Tukey's $H S D$ ) indicated that there were no significant differences between the $\mathrm{PSE}_{2: 1}$ values for the three motion conditions. However, for the high-density condition ( 1,300 points), post hoc tests revealed that the $\mathrm{PSE}_{2: 1}$ for the static (2.7), translation (2.3), and rotation (2.0) were all significantly different from each other at the .01 level.

This evidence of facilitation for both the translation and rotation conditions is consistent with the hypothesis that motion helps to resolve stereo correspondence for transparent stereo displays. As we described previously, the difficulty in processing stereo with transparent displays could result from the inability to differentiate between texture elements lying at different depths. However, by providing velocity differences for points at different depths, motion could decrease the number of mismatches in transparent displays. A simple augmentation of the nearest neighbor rule, requiring that matched points must also have the same sign or magnitude of velocity, would allow many potential false matches to be discarded.

Although both translation and rotation provided facilitation of stereo processing, the PSE 2:1 $_{1}$ values at the highdensity level for the rotation displays were significantly lower than those for the translation displays. This difference in judged depth between translation and rotation only at the high-density level indicates that SFM provided greater stereo facilitation than that provided by texture element motion alone. The additional facilitation might come from the relative depth and curvature information provided by SFM. Unlike the translation conditionwhich only provided information about whether a dot was on the front or back of the object-the rotation displays provide a gradient of relative depth that could be used to reconstruct the surface of the object. The velocities from the translation condition would have made it easy to differentiate dots on the front surface from those on the back. However, it provides no information to help distinguish between points on the same side (front or back) of the cylindrical object. Perhaps the relative depth and curvature information provided by SFM further facilitates the resolution of the stereo correspondence problem by making it easier to differentiate between texture elements on the same side of the object.

\section{GENERAL DISCUSSION}

Four principal conclusions can be reached from these results about the relationship between binocular disparity and SFM in determining perceived 3-D shape. First, there is a nonmodular, facilitative relation between these two sources of information, at least for the types of displays that we have investigated. In all of the experiments, greater depth was reported with the same disparity levels when SFM was added to a stereo display. One particularly important piece of evidence for a cooperative interaction between binocular stereopsis and SFM comes from the Experiment 1 condition in which disparity indicated a depth-to-height ratio of 3:1. The average judged depthto-height ratios were 0.52 for the static stereo version of the display and 0.50 for the monocular SFM displays. The sum of these values was considerably less than the judged ratio of 2.5 obtained for the combined stereo-SFM condition. This large difference between the separate stereo and SFM conditions, and the combined condition, cannot be explained by a mere accumulation of depth from the separate stereo and SFM processes. Consequently, we conclude that the almost fivefold increase in judged depth from the separate to the combined conditions indicates a facilitation of binocular disparity processing by SFM. Additional evidence that SFM facilitates disparity processing was found in the high-density conditions of Experiments 3 and 4 . In both of these experiments, judged depth was greater for the stereo-SFM condition than for the static stereo condition.

The second conclusion is that the depth indicated by binocular disparity affects perceived shape when both stereo and SFM information are present in a display. As we mentioned earlier, one possible way SFM and binocular disparity depth information could be combined would be through a process in which an SFM analysis provided object shape and binocular disparity resolved the orthographic ambiguity (Richards, 1985). However, data from Experiment 1 indicate that perceived depth from the combination of SFM and stereo does not solely depend on the SFM derived depth. Specifically, we found that the judged depth for stereo-SFM displays significantly increased as the amount of binocular disparity increased.

The third conclusion is that facilitation between SFM and stereopsis increases as the stereo matching problem becomes more difficult. The role of the stereo correspondence problem in the observed facilitation between binocular stereopsis and SFM was addressed in Experiments 3 and 4 . In both of these experiments, the role of the stereo correspondence process was examined by varying the texture element density for both static stereo displays and rotating stereo displays. In Experiment 3, the finding that $\mathrm{PSE}_{2: 1}$ increased with increasing density for static displays but remained approximately constant for rotating displays provides further support that SFM facilitates binocular disparity processing. Recall that increasing the 
density of texture elements in a stereo display also increases the probability that the nearest neighbor rule will provide false correspondence matches between the left and right images. Thus, the results from this experiment, confirmed in Experiment 4, indicate that the stereo correspondence process may play a central role in the observed facilitation.

The final conclusion is that the facilitation that occurs with SFM is not entirely due to the presence of opposite sign velocities on the front and back surfaces. In Experiment 1 , judged depth was greater for displays combining stereo and SFM than for displays combining stereo with translatory motion that also included opposite sign velocities. This result, however, does not necessarily imply that SFM was more effective in facilitating stereo processing. An alternative explanation is that the facilitation was equal for the two conditions, but there was an accumulation process as well, and SFM added more depth because there is more depth perceived from SFM alone than from a parallel projection of translation perpendicular to the line of sight. If the difference between the rotating and translating stereo displays was due to a difference in facilitation, we would expect the difference to increase as the stereo correspondence problem became more difficult. If the difference were due to a difference in the amount of depth perceived from SFM and translation separately, we would expect SFM to add a constant amount of depth relative to a horizontal translation, irrespective of the difficulty of the stereo correspondence problem. Experiment 4 examined the amount of disparity required to perceive a 2:1 depth-to-height ratio for stereo combined with SFM or horizontal translations, and for static stereo displays. Facilitation of binocular disparity processing was indicated for both types of motion display. However, the facilitation was significantly greater for rotation than translation stimuli. This can be seen by the fact that the high-density stereo condition $\mathrm{PSE}_{2: 1}$ was larger for the translation stimuli than for the rotation stimuli. Furthermore, the difference between these two motion conditions cannot be described by a difference between the additive combination of different monocularly specified depths. The nonparallelism of the two top lines in Figure 6 for 2 of the 3 subjects demonstrates that the rotation displays did not simply add a constant to the depth seen with the translation displays. This result indicates that, although some of the facilitation of stereo reported here can be attributed to the presence of opposite sign velocities on the front and back surfaces, additional facilitation results from the presence of SFM.

The results from the translation stereo displays also provide evidence that all of the observed facilitation cannot be explained simply as the difference between motion disparity and position disparity (Regan et al., 1979). Because the texture elements in the translation condition do not move in depth, they do not possess motion disparity. Thus, the fact that these displays without motion disparity exhibit facilitation indicates that motion disparity is not a necessary condition for stereo facilitation from motion.
Furthermore, the evidence from Experiments 3 and 4 supporting the importance of the correspondence problem for stereo facilitation does not favor a motion disparity interpretation. The depth-from-motion disparity process, as described by Regan et al. (1979), compares the image velocities of the texture elements in the left and right eyes after the matching process has been completed. The image velocities are not used to resolve correspondence in this process, and thus there is no clear reason for motion disparity to provide greater facilitation of stereo processing for high- rather than low-density displays.

Although the data presented here indicate that SFM and binocular stereopsis interact cooperatively, it is important to note that cooperativity and accumulation are not mutually exclusive. One possible formulation of the integration of depth from stereopsis and SFM is a two-step process in which SFM first facilitates the resolution of the stereo correspondence problem, and then the depths derived from SFM and stereopsis are combined in an accumulative manner to achieve a final percept. This weak modularity architecture would preserve, to some degree, the autonomy of the individual depth-perception processes and enable them to operate in the absence of additional information, as demonstrated by previous research. But it would also allow for various processes to share information, and thus provide more consistent and error-free representations of the shape and depth of objects in the environment.

A particularly interesting issue concerns the specific mechanism by which SFM facilitates binocular disparity processing. In Experiment 2, we examined the hypothesis that motion and SFM facilitate stereo processing by decreasing the time needed to resolve correspondence. However, for the stimulus durations presented, no evidence of temporal facilitation was found. This result was consistent with the fact that, in Experiment 1, a significant difference was observed between the static stereo and SFM-stereo conditions, even with an unlimited viewing time. As we stated earlier, the data from Experiments 3 and 4 provide evidence supporting the hypothesis that SFM helps to resolve the stereo correspondence problem. In addition, the difference between the PSE $2: 1$ for the SFM-stereo and translation stereo conditions in Experiment 4 make it clear that not all of the facilitation can be explained by opposite sign velocity of texture elements on the front and back surfaces. One possibility would be that SFM provides information such as surface curvature that could be used to constrain the number of potential matches and thus aid the correspondence process. Such a scheme would be more flexible than a fixed constraint process, because it could potentially alter its matching constraints on the basis of properties of the object specified by other sources (SFM, in this case).

Another question of interest is the relative importance or weight of SFM and binocular disparity when the two sources of information are combined. It seems likely that these weights would not remain constant, but vary according to the situation and salience of the information under 
observation (Landy \& Maloney, 1990). If this were so, it would be particularly interesting to uncover the factors that determine the relative importance or weighting of different sources of visual information when they are combined.

The visual properties of objects in the environment are represented by numerous sources of information. A complete understanding of visual perception will thus include an explanation of how this multitude of information is combined into a unified percept. The research presented here provides information about the integration of visual information from two well-studied sources: binocular stereopsis and structure from motion. The results from these experiments indicate that the processing of depth information from SFM and binocular disparity is not strictly modular, and thus theories of combining visual information that assume strong modularity or independence cannot accurately characterize all instances of depth perception from multiple sources.

\section{REFERENCES}

AKerstrom, R. A., \& TodD, J. T. (1988). The perception of stereoscopic transparency. Perception \& Psychophysics, 44, 421-432.

andersen, G. J., \& Braunstein, M. L. (1983). Dynamic occlusion in the perception of rotation in depth. Perception \& Psychophysics, 34, 356-362.

ANDERSON, N. H. (1981). Foundations of information integration theory. New York: Academic Press.

ANDERSON, N. H. (1982). Methods of information integration theory. New York: Academic Press.

Braunstein, M. L. (1976). Depth perception through motion. New York: Academic Press.

Braunstein, M. L., Andersen, G. J., Rouse, M. W., \& Tittle, J. S. (1986). Recovering viewer-centered depth from disparity, occlusion, and velocity gradients. Perception \& Psychophysics, 40, 216-224.

Bruno, N., \& Cutting, J. E. (1988). Minimodularity and the perception of layout. Joumal of Experimental Psychology: General, 117, $161-170$.

Bülthoff, H., \& Mallott, H. (1988). Integration of depth modules: Stereo and shading. Journal of the Optical Society of America A, $\mathbf{5}$, 1749-1758.

Dosher, B., SPERling, G., \& WURSt, S. (1986). Tradeoffs between stereopsis and proximity luminance covariance as determinants of perceived 3D structure. Vision Research, 26, 973-990.

EPSTEIN, W. (1968). Modification of the disparity-depth relationship as a result of exposure to conflicting cues. American Journal of Psychology, 81, 189-197.

Fiśher, S. K., Ebenholtz, M. S. (1986). Does perceptual adaptation to telestereoscopically enhanced depth depend on the recalibration of binocular disparity? Perception \& Psychophysics, 40, 101-109.

FoDor, J. (1983). The modularity of mind. Cambridge, MA: MIT Press.

GOGEL, W. (1960). The perception of depth interval with binocular disparity cues. The Journal of Psychology, 50, 257-269.

Johnston, E. B., LANDY, M. S., Cumming, B. G., Maloney, L. T. (1991). Integration of stereo and motion shape cues. Investigative Ophthalmology \& Visual Science, 32(Suppl. 4), 1180.

LANDY, M. S., \& MALONEY, L. T. (1990). A statistical framework for combination of consonant depth cues. Investigative Ophthalmology \& Visual Science, 31(Suppl. 4), 173.

LAPPIN, J. S., \& Love, S. R. (1992). Planar motion permits perception of metric structure in stereopsis. Perception \& Psychophysics, 51, 86-102.

MarR, D., \& Poggio, T. (1976). Cooperative computation of stereo disparity. Science, 194, 283-287.
Nawrot, M., \& BLAKE, R. (1991). The interplay between stereopsis and structure from motion. Perception \& Psychophysics, 49, 230-244.

OGLE, K. N. (1953). Precision and validity of stereoscopic depth perception from double images. Joumal of the Optical Society of America, $43,906-913$

ONo, H., \& Comerford, J. (1977). Stereoscopic depth constancy. In W. Epstein (Ed.), Stability and constancy in visual perception (pp. 375 419). New York: Wiley.

Pong, T. C., Kenner, M. A., \& Otis, J. (1989). Stereo/Motion cues in preattentive vision processing: Some experiments with random dot stereographic image sequences. Proceedings of the IEEE Workshop on Visual Motion, 314-320.

Prazdny, K. (1985). Detection of binocular disparities. Biological Cybermetics, 52, 93-99.

Regan, D., Beverley, K. I., \& Cynader, M. (1979). Stereoscopic subsystems for position in depth and for motion in depth. Proceedings of the Royal Society of London, B 204, 485-501.

RicHARDS, W. (1985). Structure from stereo and motion. Journal of the Optical Society of America A, 2, 343-349.

Rogers, B. J., Collett, T. S. (1989). The appearance of surfaces specified by motion parallax and binocular disparity. Quarterly Journal of Psychology, 41A, 697-717.

Rouse, M. W., Tittle, J. S., \& Braunstein, M. L. (1989). Stereoscopic depth perception by static stereo-deficient observers in dynamic displays with constant and changing disparity. Optometry \& Vision Science, 66, 355-362.

TitTle, J. S., \& Braunstein, M. L. (1989). The interaction of binocular disparity and structure-from-motion in determining perceived relative depth. Investigative Ophthalmology \& Visual Science, 30 (Suppl. 4), 263.

Tittle, J. S., \& Braunstein, M. L. (1991). Shape perception from binocular disparity and structure-from-motion. In P. S. Schenker (Ed.), Proceedings of the Sociery of Photo-optical Instrumentation Engineers: Sensor Fusion III: 3-D Perception and Recognition. Bellingham: WA: Society of Photo-optical Instrumentation Engineers.

Wallach, H., Moore, M. E., \& Davidson, L. (1963). Modification of stereoscopic depth-perception. American Journal of Psychology, 76, 191-204.

Wallach, H., \& O'ConNell, D. N. (1953). The kinetic depth effect. Journal of Experimental Psychology, 45, 205-217.

Wallach, H., Zuckerman, C. (1962). The constancy of stereoscopic depth. American Journal of Psychology, 76, 404-412.

\section{NOTES}

1. In this paper, we limit the definition of structure from motion to include only parallel projections of pure rotation.

2. Motion disparity refers to the different velocities in the left and right retinal images that occur for any motion of a texture element in 3-D space, except for translation along a line perpendicular to the viewing direction. The dots in our rotation displays had different velocities in the left and right images, but the dots in our translation displays had the same velocity in both images and thus did not have motion disparity.

3. It is possible that duration (in this experiment and Experiment 3 ) or density (in Experiments 3 and 4) affected the perceived height of the cylinders, but we have no indication of such an effect. It seems unlikely that perceived height would have been affected differently by duration and density in the different motion conditions.

4. The nearest neighbor criterion is a matching rule that requires a point in one image to be matched with the point in the other image that comes closest to having the same location. More complex algorithms have been proposed for solving the stereo correspondence problem involving either cooperative (Prazdny, 1985) or inhibitory (Marr \& Poggio, 1976) interactions among neighboring units.

(Manuscript received February 17, 1992; revision accepted for publication January 13, 1993.) 